\title{
ECG Simulations with Realistic Human Membrane, Heart, and Torso Models
}

\author{
Mark Potse, Bruno Dubé, and Ramesh M. Gulrajani \\ Institute of Biomedical Engineering, Université de Montréal, Montréal, Québec, Canada
}

\begin{abstract}
We implemented a recent model of the human ventricular cell membrane in our existing human heart model comprising 12 million cells. Using an inhomogeneous torso model, a normal ECG was simulated, as well as ECGs for a membrane model with modified transient outward current. We also investigated the effect of cellular coupling in the heart on the action potentials, and show that electrotonic coupling results in a continuous transmural distribution of action potential durations, despite the presence of only three different inherent cell types. 25th Annual International Conference of the IEEE EMBS, Cancun, Mexico, September 2003, session 1.1.3.
\end{abstract}

Keywords-ECG, heart model, membrane model, simulation

\section{INTRODUCTION}

Modeling of the electrical activation of the heart allows us to test if our knowledge of small-scale phenomena, such as the behavior of the cardiac membrane, suffices to explain largescale phenomena, such as the ECG. If our models prove to be capable of this, we may also use them to predict the effect that modifications on the cellular level have on the ECG. One may, for example, modify a model of the cell membrane so as to imitate the implications of a genetic defect, and observe the specific changes in the ECG. Such prediction can help us to improve diagnostic methods.

We used a human heart model consisting of 12 million cells. Each cell was represented by a model of the human ventricular cell membrane described recently by Bernus et al. [1]. This membrane model is a compromise between the accuracy obtained by its ancestor, the Priebe-Beuckelmann model [2], and the efficiency required by a large-scale heart model. In this paper we describe our attempts to simulate a normal ECG using this model implanted in a realistic inhomogeneous model of the human torso. We also describe the effects of electrotonic coupling in the heart on action potential (AP) durations, and the effects of blocking the transient outward current on the ECG.

\section{Methods}

\section{A. The model}

Our starting point was a finite-difference heart model incorporating 0.2 million cells that was based on CT data of a human heart obtained at autopsy [3]. The finite-difference grid was refined by a factor of 4 in each dimension in order to realise sufficiently accurate representations of the propagating activation front [4]. We thus obtained a 12-million-cell heart model. The model is anisotropic with rotating fiber direction, and incorporates a representation of the specialized conduction system using early activation times as published by Durrer et al. [5].

Ionic currents were computed using Bernus' model of human ventricular cells [1]. This model includes 3 different cell types: endocardial, midmyocardial ( $\mathrm{M}$ cell) and epicardial. We modified one of the parameters of the model (the potassium conductance, $g_{\mathrm{K}}$, see Table I), in order to obtain realistic T-waves. Propagation of activation was governed by a reaction-diffusion equation that related the spatial distribution of the membrane potential to its temporal change [4]:

$$
\frac{\partial V_{\mathrm{m}}}{\partial t}=\frac{1}{\beta C_{\mathrm{m}}}\left[\frac{\xi}{1+\xi} \nabla \cdot\left(G_{\mathrm{e}} \nabla V_{\mathrm{m}}\right)-\beta I_{\text {ion }}+\frac{\beta}{1+\xi} I_{\text {stim }}\right]
$$

where $V_{\mathrm{m}}$ is the membrane potential, $C_{\mathrm{m}}$ the membrane capacitance, $\beta$ the surface-to-volume ratio of the ventricular cell; $\xi$ the ratio of intracellular and extracellular conductivity (assumed fixed), $G_{\mathrm{e}}$ the conductivity tensor of the extracellular domain, $I_{\text {ion }}$ the ionic current provided by the membrane model, and $I_{\text {stim }}$ an optional stimulation current. This equation was solved with an initial time step of $25 \mu \mathrm{s}$; once all cells had completed their upstrokes, the time step was increased to $50 \mu \mathrm{s}$.

The surface ECG was computed using a multiple dipole representation derived from the spatial gradient of $V_{\mathrm{m}}$, and an inhomogeneous human torso model [3]. Myocardial anisotropy was ignored for ECG computation.

Simulation of propagation was performed on 16 processors of a 64-processor SGI Origin 2000 computer $(400 \mathrm{MHz}$, 2 FLOPS per cycle). This computer consists of 32 nodes, each containing 2 processors and $2 \mathrm{~GB}$ of memory. Although its memory is decentralized, the system employs a single memory image. The programs were written in $\mathrm{C}$, and parallelized using OpenMP directives by assigning a part of the model to each processor: since the model had approximately 12 million cells, each processor handled about 0.75 million cells. The parallel tasks were synchronized at each time step because the spatial distribution of $V_{\mathrm{m}}^{t}$ is involved in the computation of

TABLE I

POTASSIUM CONDUCTANCES OF THE ORIGINAL AND MODIFIED MEMBRANE MODELS

\begin{tabular}{lccl} 
& Bernus & current & purpose \\
\hline$g_{\text {K,epi }}$ & 0.018 & 0.036 & earlier \& larger T-wave \\
$g_{\mathrm{K}, \mathrm{M}}$ & 0.013 & 0.013 & \\
$g_{\mathrm{K}, \text { endo }}$ & 0.019 & 0.030 & earlier T-wave
\end{tabular}



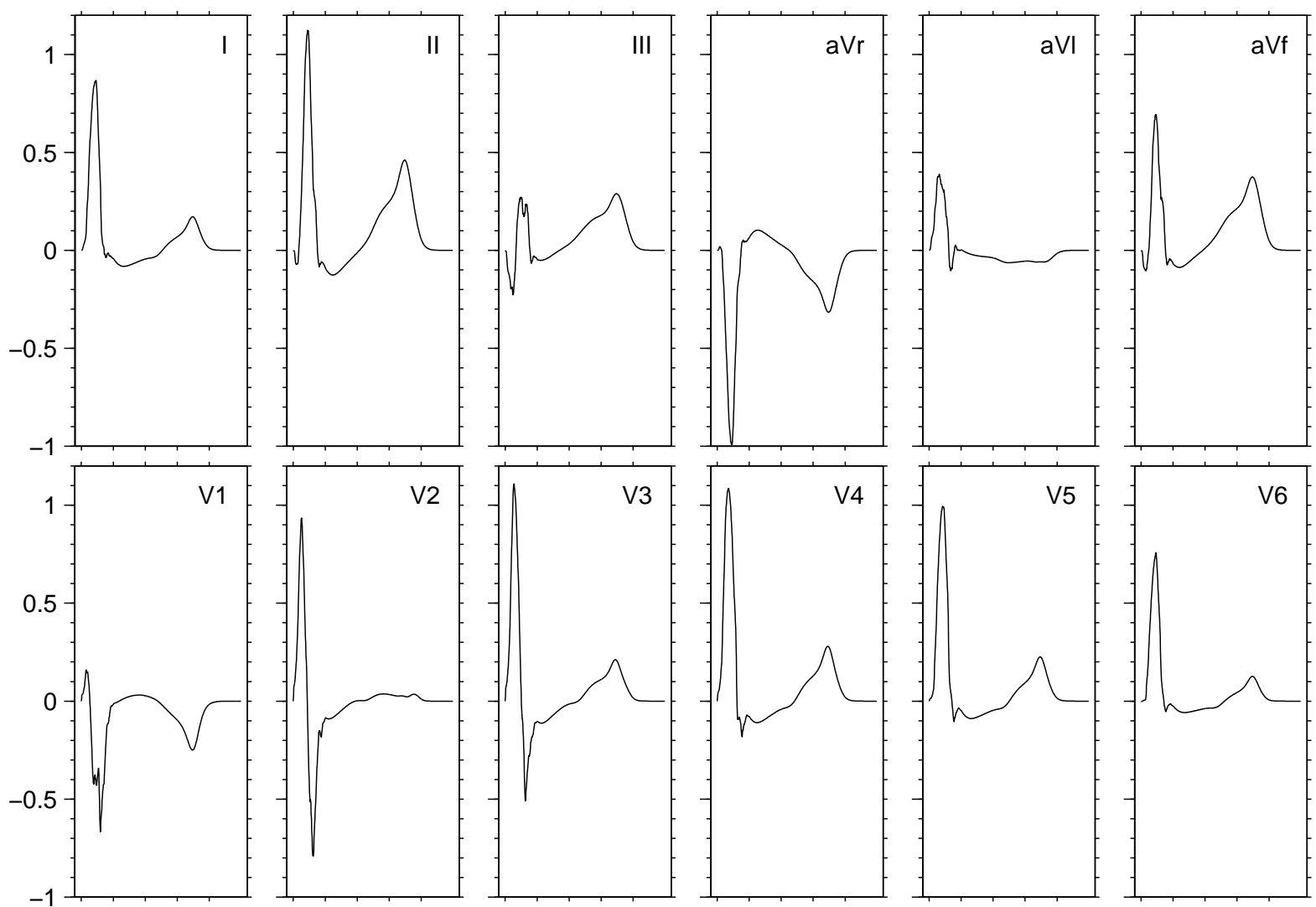

Fig. 1. Standard 12-lead ECG of sinus rhythm under normal circumstances. Tick marks along the horizontal axes indicate intervals of 100 ms. The vertical axes are in $\mathrm{mV}$.

$V_{\mathrm{m}}^{t+1}$. This necessitates interprocessor communication. However, interprocessor communication was limited to the membrane potentials of cells on the boundaries of the processors' domains. Thus, the amount of data transported between processors was very small compared to the amount of data handled within each processor. Due to the shared memory image, no effort was needed to organize this interprocessor communication. However, care was taken to place the memory used by each processor on the processor's own node, since this minimizes memory access times. Simulation of propagation took approximately 5 hours for a single heart beat of $500 \mathrm{~ms}$. Computation of ECGs from the action potentials thus obtained took only a few minutes.

\section{B. Simulations}

Normal propagation and a normal ECG were simulated using our current values of all model components, i.e. incorporating our changed $g_{\mathrm{K}}$ values described above.

In order to study the effect of cellular coupling, the action potentials generated during activation by cells across the ventricular wall were compared to the action potentials of these same cells when isolated.

We also made an effort to verify the effects of the transient outward current on $\mathbf{J}$ waves in the ECG. $\mathbf{J}$ waves are small positive deflections observed right at the end of QRS, and have been attributed to the presence of the transient outward current in epicardial cells [6], [7], [8], [9]. These assertions are easy to verify in our model by simply modifying the conductance $g_{\text {to }}$, which controls the transient outward current, in endocardial, $\mathrm{M}$, and epicardial cells.

\section{RESULTS}

We had to modify the values of potassium conductance from those used by Bernus et al. [1] in order to obtain realistic T-waves. Original and modified conductances are shown in Table I. The resulting ECG is shown in Fig. 1.

A continuous transmural variation in AP duration and shape was observed, despite the presence of only 3 discrete inherent cell types (Fig. 2). Coupling affects M cells the most, reducing their AP durations by about $50 \mathrm{~ms}$ compared to those of isolated M cells. Endocardial and epicardial AP durations are increased by approximately $20 \mathrm{~ms}$ due to coupling.

Very small $\mathbf{J}$ waves could be discerned in the left precordial leads of our normal ECG (Fig. 1). In most leads, a small $\mathrm{S}-\mathrm{T}$ depression was present. These phenomena disappeared when the transient outward current $\left(I_{\mathrm{to}}\right)$ was blocked for all cell types by setting its conductance, $g_{\text {to }}$, to zero. Subsequent introduction of a normal $g_{\text {to }}$ value for M cells led to a $0.1-$ 

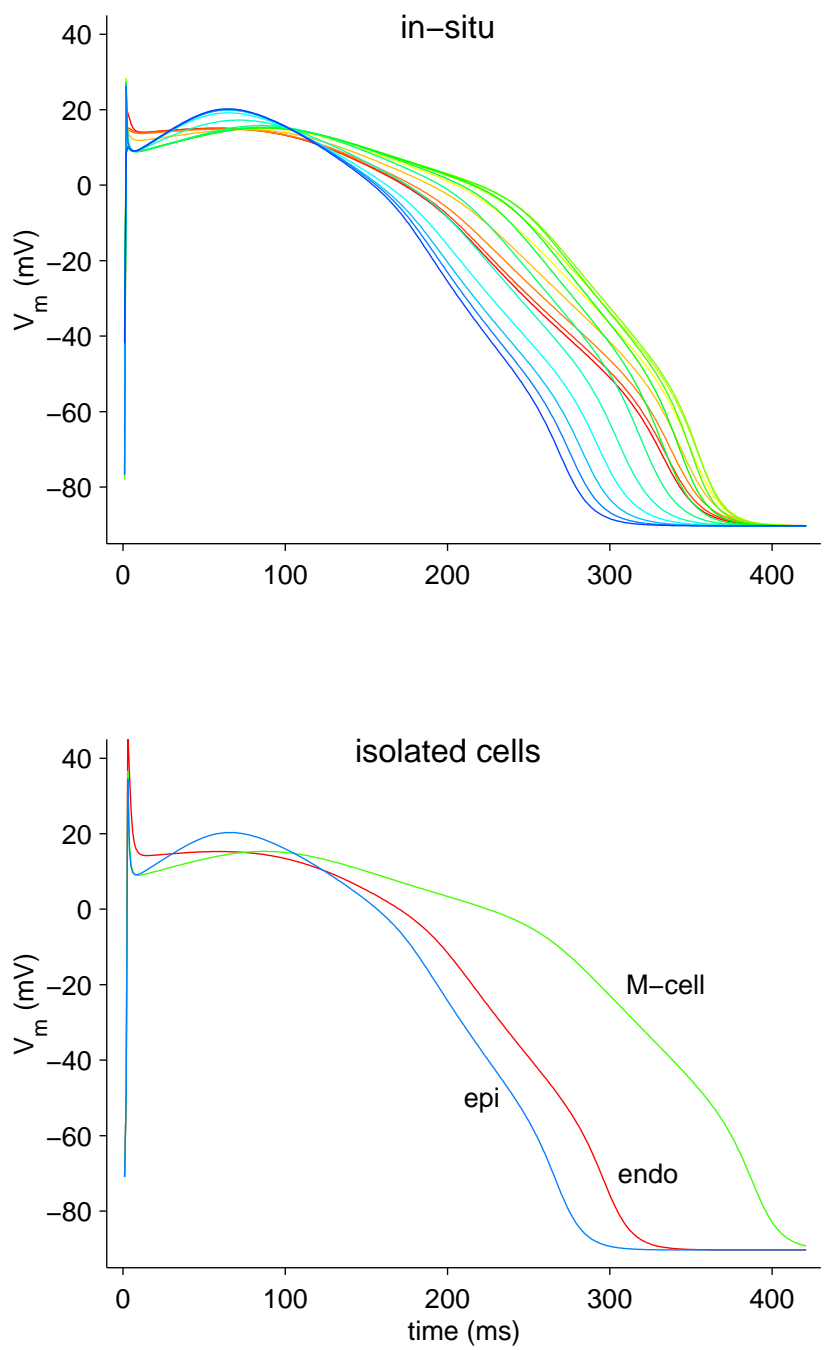

Fig. 2. Action potentials of a line of cells that spans the free wall of the left ventricle from endocardium to epicardium (red for endocardium, green for M-cells, and blue for epicardium with a gradual shift in hue). Panel A shows the action potentials obtained in-situ, in the full heart model, aligned on the AP upstroke. Panel B shows the inherent AP shapes that are obtained when these same cells are isolated. Since discrete cell types were used, only three traces are present.

$0.2 \mathrm{mV}$ depression of the ST segment, but not to J waves.

$\mathrm{J}$ waves were only elicited with normal or increased $g_{\text {to }}$ values for epicardial cells. Increasing $g_{\text {to }}$ for epicardial cells led to an enhancement of the J waves (Fig. 3). The $g_{\text {to }}$ of M cells had little influence on the $\mathrm{J}$ wave.

\section{Discussion}

Simulation of a surface ECG based on a realistic model of the cell membrane and realistic models of the heart and the torso anatomy can be performed in a few hours on a parallel computer with a modest number of processors.

In order to obtain normal T-waves, we had to modify the AP durations of the various cell types. We did this by changing the $g_{\mathrm{K}}$ values. This may indicate that measured values of
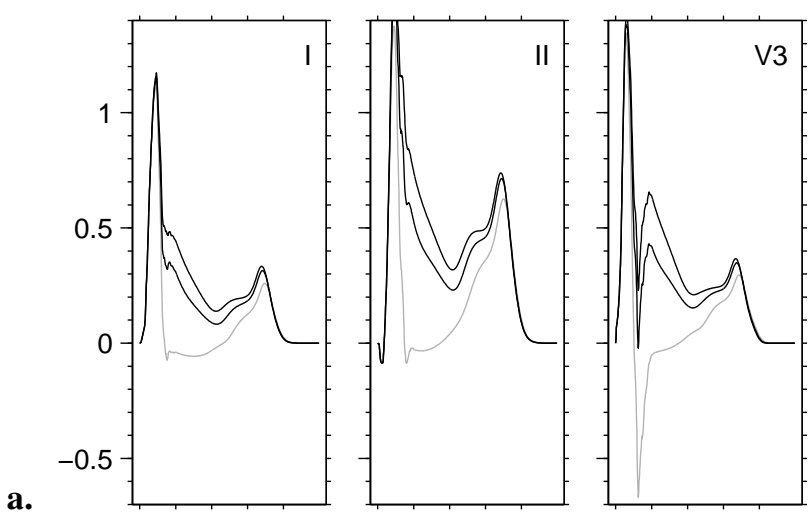

\section{b.}
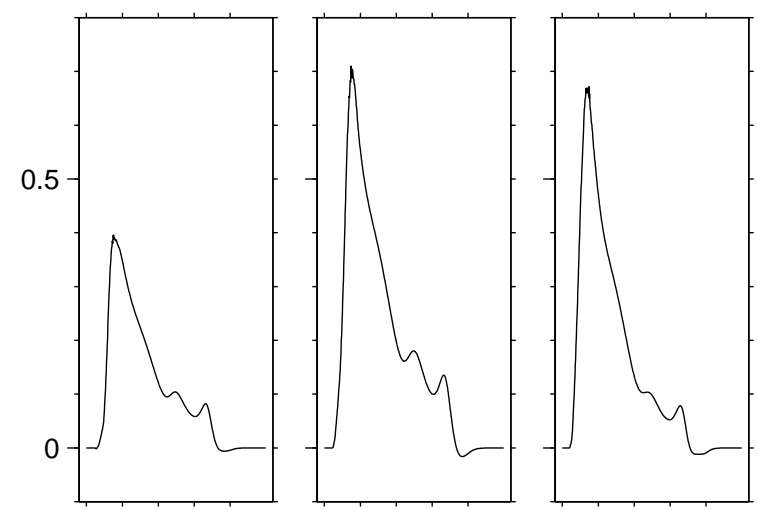

Fig. 3. Panel a: Selected standard leads of sinus rhythm, with normal and enhanced $g_{\text {to }}$ (black) as well as zero $g_{\text {to }}$ (grey) for epicardial cells. Endocardial and midmyocardial $g_{\text {to }}$ is zero. Panel b: Isolated J waves, obtained as the difference between the leads with normal and zero epicardial $g_{\text {to }}$. In both panels, tick marks along the horizontal axes indicate intervals of $100 \mathrm{~ms}$. The vertical axes are in $\mathrm{mV}$.

model parameters such as $g_{\mathrm{K}}$ are incorrect, but it is equally possible that the discrepancy is caused by an inadequacy in our methods. For example, the stratification of cell types has an important effect on the amplitude of T-waves. Thus, while three different cell types have been identified in human ventricular myocardium, the actual thickness of the three layers is not accurately known, and we may have chosen them incorrectly.

Cellular coupling has a large effect on AP duration. This should be taken into account when comparing in-situ with isolated-cell measurements, as others have pointed out before [9], [10], [11].

$\mathbf{J}$ waves are rare in humans but common in some animal species, including dogs [6], [7]. J waves were previously simulated by Gima and Rudy using a one-dimensional model with Luo-Rudy type II kinetics and a pseudo-ECG that is somewhat representative for left precordial leads [7]. We have confirmed their finding of $I_{\mathrm{to}}$-dependence of the $\mathrm{J}$ wave using a model that was created specifically for human ventricular cells [1]. Only with $g_{\text {to }}$ present in epicardial cells were $\mathbf{J}$ waves present. The presence of $g_{\text {to }}$ in endocardial and M cells did not result in $\mathrm{J}$ waves per se. In addition to confirming the results ob- 
tained by Gima and Rudy we have computed a realistic standard ECG, and by subtraction of leads shown the actual shape of the $\mathbf{J}$ wave. From Fig. 3 it is clear that the onset of this wave is masked by the QRS complex. This is not due to the amplitude of the QRS, but merely to its steep slope, which makes it difficult for the human eye to estimate the difference in potential at one time instance between two waveforms. Only at "high paper speed," inspection of the wave shapes themselves would allow recognition of relatively small waves superposed on a QRS complex.

\section{ACKNOWLEDGMENT}

The authors thank the Réseau québécois de calcul de haute performance (RQCHP) for access to their computer systems, and Dr. Jacques Richer for his expert assistance with optimization of the code. This work was supported by the Natural Sciences and Engineering Research Council of Canada. M. Potse was supported by FCAR, Québec, and The Netherlands Organization for Scientific Research (NWO).

\section{REFERENCES}

[1] O. Bernus, R. Wilders, C. W. Zemlin, H. Verschelde, and A. V. Panfilov, "A computationally efficient electrophysiological model of human ventricular cells," Am. J. Physiol. Heart Circ. Physiol., vol. 282, pp. H2296-H2308, 2002.

[2] L. Priebe and D. J. Beuckelmann, "Simulation study of cellular electric properties in heart failure," Circ. Res., vol. 82, pp. 1206-1223, 1998.

[3] M. Lorange and R. M. Gulrajani, "A computer heart model incorporating anisotropic propagation: I. Model construction and simulation of normal activation," J. Electrocardiol., vol. 26, pp. 245-61, Oct. 1993.

[4] M.-C. Trudel, R. M. Gulrajani, and L. J. Leon, "Simulation of propagation in a realistic-geometry computer heart model with parallel processing," in CDROM Proc., 23rd Annual International Conf. IEEE Eng. Med. Biol. Soc., 2001.

[5] D. Durrer, R. T. van Dam, G. E. Freud, M. J. Janse, F. L. Meijler, and R. C. Arzbaecher, "Total excitation of the isolated human heart," Circulation, vol. 41, pp. 899-912, June 1970.

[6] G.-X. Yan and C. Antzelevitch, "Cellular basis for the electrocardiographic J wave," Circulation, vol. 93, pp. 372-379, 1996.

[7] K. Gima and Y. Rudy, "Ionic current basis of electrocardiographic waveforms; A model study," Circ. Res., vol. 90, pp. 889-896, 2002.

[8] G.-R. Li, J. Feng, L. Yue, and M. Carrier, "Transmural heterogeneity of action potentials and $I_{\text {to } 1}$ isolated from the human right ventricle," Am. J. Physiol Heart Circ. Physiol., vol. 275, pp. H369-H377, 1998.

[9] E. Drouin, F. Charpentier, C. Gauthier, K. Laurent, and H. Le Marec, "Electrophysiologic characteristics of cells spanning the left ventricular wall of human heart: Evidence for presence of M cells," J. Am. Coll. Cardiol., vol. 26, no. 1, pp. 185-192, 1995.

[10] E. P. Anyukhovsky, E. A. Sosunov, R. Z. Gainullin, and M. R. Rosen, "The controversial M cell," J. Cardiovasc. Electrophysiol., vol. 10, pp. 244-260, 1999.

[11] C. Antzelevitch, W. Shimizu, G.-X. Yan, S. Sicouri, J. Weissenburger, V. V. Nesterenko, A. Burashnikov, J. Di Diego, J. Saffitz, and G. P. Thomas, "The M cell: Its contribution to the ECG and to normal and abnormal electrical function of the heart," J. Cardiovasc. Electrophysiol., vol. 10, pp. 1124-1152, 1999. 